\title{
Office of Environmental Policy and Assistance. RCRA/CERCLA Division (EH-413) Guidance Document Publications List
} August 1995

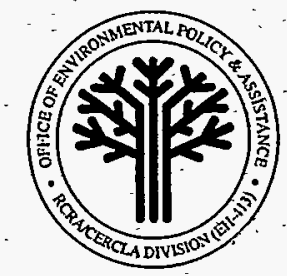

\section{RCRA Guidance Manuals}

Obtaining Variances from the Treatment Standard's of the RCRA Land Disposal Restrictions. .DOEIEGD-001-0590, May 1990 Closure of Hazardous and Mixed Waste Management Units at DOE Facilities DOEJEGD(RCRA)-002/0690, June 1990 New LDR for Contaminated Soil and Debris and Newly Identified Toxicity Characteristic Organic Wastes . (Not numbered), May 1992 Regulated Underground Storage Tanks (USTs) Guidance DOEIEH-231-004/0191, June 1992 Preparation of RCRA Contingency Plans DOEJEH-0274, July 1992 Definition of Solid and Hazardous Waste DOEJEH-273, August 1992 Preparation of RCRA Waste Analysis Plans (Interim faxidance) DOEIEH-0306, March 1993 LDR Program Overview RCRA Correction Action Program Gutude Management of Hazardous Waste Containers and Conathe Storage Afés Under RCRA

\section{RCRA Infomation Briefs}

Requirements for Recycling of fazatdous Waste DOEJEH-231-001/0990 The Small-Scale Treatlabillixy Study satriple Exemption DOOEJEH-231-002/0191 Questions and Answers on the Rend Tox leity Characteristic DOE/EH-231-003/0291 The Mixture Rule under the Resource conservation ard Recovent Ad DOEIEH-231-005/0991 RCRA Contingency Plans and Emergency Procedtites DOE/EH-231-006/0991 Overview of the Identification of Hazardous waste under RCRA DOEIEH-231-007/1291 Identification of Certain RCRA Waste-the F-Spent Solvent, $P$, and bet istutgs DOE/EH-231-008/1291 RCRA Closure and Post-Closure Plans DOEE/EH-231-009/1291 RCRA Clean Closure Equivalency Demonstrations DOEJEH-231-010/1291 Waste Management and the Land Disposal Restriction Storage Pxotibition DOE/EH-231-011/0592 Loss of Interim Status (LOIS) under RCRA DOE/EH-231-0181/0992

RCRAAir Emission Standards for Hazardous Waste Treatment, Storage, athe bisposal facility

(TSDF) Equipment Leaks

DOE/EH-231-019/0193

RCRAAir Emission Standards for Hazardous Waste Treatment, Storage and Disposaf Facility

(TSDF) Process Vents

DOE/EH-231-020/0193

RCRA Post-Closure Permits

DOE/EH-231-021/0293

Identifying and Classifying an UST

DOE/EH-231-012a/0593

Excluded USTs

DOEJEH-231-012b/0593

Deferred USTs DOE/EH-231-012c/0593

Requirements for Satellite Accumulation Areas DOE/EH-231-026/0593 


\section{RCRA Information Briefs (continued)}

Management of Corrective Action Wastes. Pursuant to Proposed Subpart S DOEJEH-231-029/0295

Exclusions and Exemptions from RCRAHazardous Waste Regulations DOEJEH-231-034/0593 RCRA Corrective Action Variances from Subpart F Requireménts DOEJEH-231-025/0693

The 'Derived-from' Rule under the Resource Conservation-and-Recovery Act (RCRA) .DOE/EH-231-035/0693 RCRA Corrective Action Permit Requirements and Modifications under Subpart F Regulations .DOE/EH-231-022/0793 RCRA Corrective Action Permit-Requirements and Modifications, under Subpart S RuleDOEJEH-231-023/0793 RCRA Corrective Action Interim Measures under the Proposed Subpart S Rule DOE/EH-231-024/0793 RCRA Hazardous Waste Container Labeling, Marking, and Placarding Requirements DOE/EH-231-031/0793 Closure of Municipal Solid Waste Landfills (MSWLFs) .DOEIEH-231-036/0793 Inspections of RCRA Container Storage Areas .DOE/EH-231-033-0793 Delay of Closure for RCRA Hazardous Waste Management Fackikg



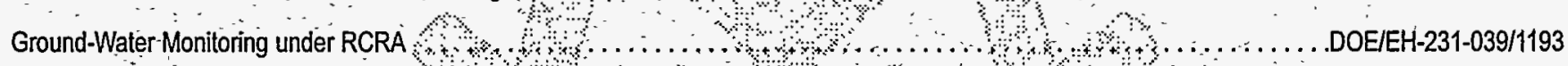

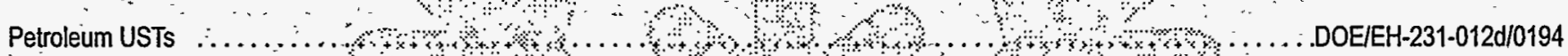
Hazardous Substance USTS Corrective Action Management Untls and Temporany Unís $\ldots \ldots \ldots$. . . DOE/EH-231-043/0394

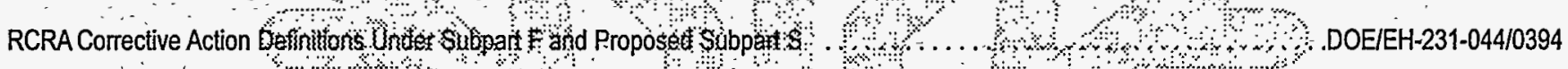

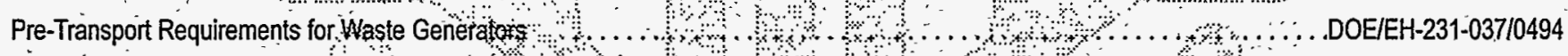

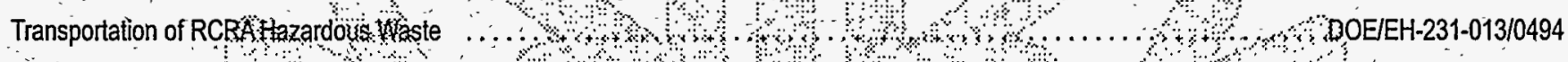
Manifest Requirements

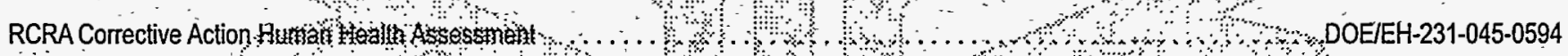

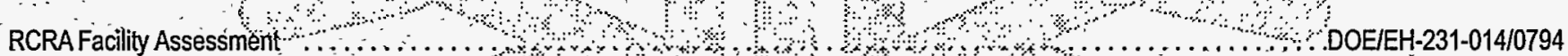

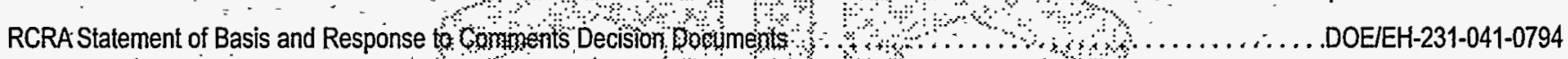

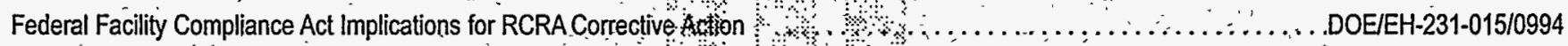

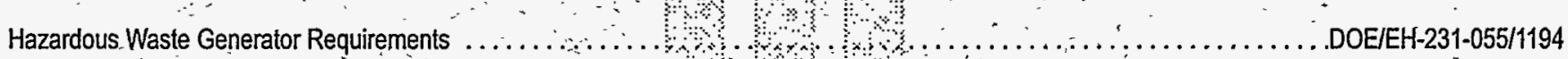

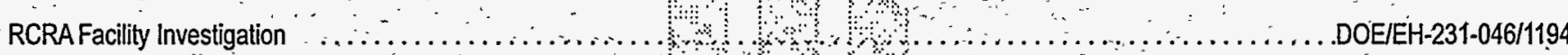

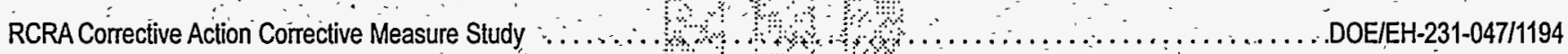
RCRA Corrective Action Corrective Measures Implementation General Facility Standard Requirements Ignitable, Corrosive, Reactive and Incompatible Wastes Und DOE/EH-231-043/1294 RCRA Corrective Action and Closure DOEE/EH-231-054/1294 DOEIEH-231-051/0295 RCRA Facility Stabilization Initiative .DOEJEH-231:016/0295 RCRA Corrective Action Workplans .DOEJEH-231-030/0295 RCRA Corrective Action: Action Levels and Media Cleanup Standàrds DOE/EH-231-050/0295 Transfer of Environmental Permits After the Sale or Transfer of DOE Property DOEJEH-231-061/0895 Regulatory Requirements Affecting Disposal of Abestos-Containing Waste DOEJEH-231-062/0895 


\section{RCRA Regulatory Bulletins}

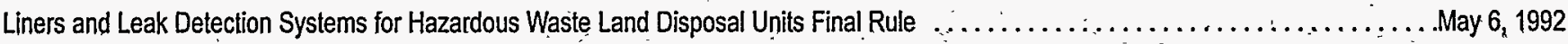

Hazardous Debris Case-by-Case Capacity Variance July 15,1992

Amendments to Interim Status Downgradient Groundwater Monitoring Standards at Hazardous Waste Facilities Sepfember 23, 1992

Identification and Listing of Hazardous Waste - Used Oil Final Rule Issued

December 31, 1992

CAMU/TU Final Rule lssued . .

May 12, 1993

Liquids in Landfills - Final Rule Issued

July 1,1993

Renewal of Hazardous Debris Case-by-Case Capacity Variance

July 28, 1993

Reportable Quantity Adjustments for Lead Metal, Lead Compounds,

Lead-containing Hazardous Wastes, and Methyl Isocyanate

September 15, 1993

Used Oil Final Rule and Correction Notices Issued

.October 31, 1993

Hazardous Waste Treatability Studies Sample Exclusion - Revișed Rule Issued

.October 20,1994

\section{CERCLA Gutian Hanuals}

Catalog of CERCLAApplicable or Relevant and Appropqate Requiremen (ARARS)

"Quick Reference Fact Sheets"

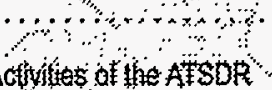

.EGD (CERCLA)-002/0790, July 1990

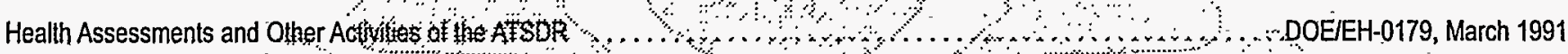

Emergency Planning and Commatily Right-to-Know Adt (EPCRA)

DOEJEH-0181P, March 1991

Superfund Program Analysig-iessons learned

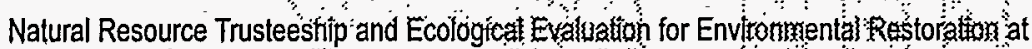

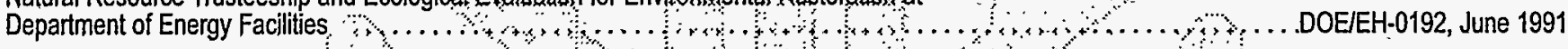
Integrated Risk Informatter Systep

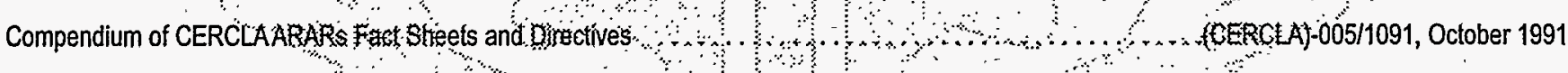

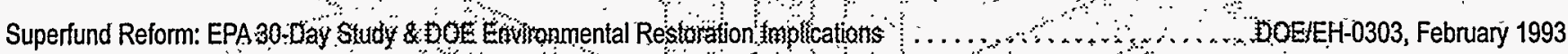

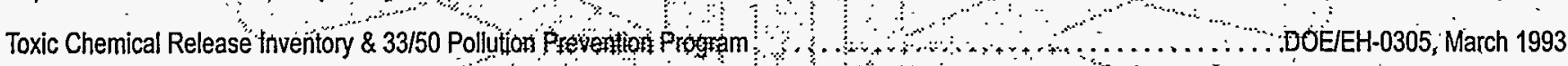

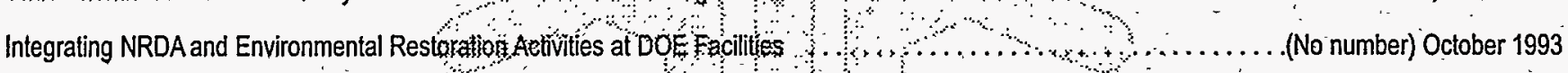
CERCLA Site Assessment Qs \& As

A Guide to CERCLA Site Assessments

Hazardous Substance Release Reporting under CERCLA, EPCRA 304 , \& bOE

Emergency Management System/Occurrence Reporting Requirethents

DOEJEH-0383, June 1994

Incorporating Ecological Risk Assessment into RI/FS Work Plans

DOEJEH-0391; June 1994 CERCLA Site Assessment Workbook Volume I and Video .DOEJEH-23-0002(a),August 1994 CERCLA Site Assessment Workbook Volume II and Video DOE/EH-23-0002(b), August 1994

Reporting Continuous Releases of Hazardous and Extremely Hazartous sutstances

Under CERCLA and EPCRA

.DOE/EH-0441, January 1995

CERCLA 103 and EPCRA§304 Release Notification Requirements Update

DOE/EH-0447, January 1995

CERCLA Removal Actions DOE/EH-0435, September 1994

Streamlined Approach for Environmental Restoration (SAFER) Pilot Project .DOE/EH-0444, January 10, 1995

RIIFS Process, Elements and Techniques Guidance DOEIEH 94007658, December 21, 1993

Glossary of CERCLA-Related Terms and Acronyms

DOEIEHO219, October 1994

CERCLABaseline Risk Assessment Reference Manual

.DOE/EH-0484, March 1995 
- Post Office, if unable to deliver please return to:

Reisa Kall (EH-413)

U.S. Department of Energy

1000 Independence Ave., S.W.

Róom GA076

Washington, B.C. '20585

(202) $586-6374$

ADDRESS CORRECTION REQUESTED



This Publication List is

printed on recycled paper 
Reporting Releases of Hazardous Substances under CERCLAand EPCRA

.DOEIEH-231-001/0490

Complying with Land Disposal Restrictions (LDR) for CERCĹARemedial Actions

Involving Contaminated Soil and Debris

DOE/EH-231-002/0191

"Hazardous" Terminology .DOE/EH-231-003/0191

"Toxic" Terminology DOEJEH-231-004/0191

Natural Resource Damage Assessment: Preassessment Screening and Integration with CERCLA Ecological Evaluations DOEJEH-231-008/1091

Federal Agency Hazardous Waste Compliance Docket DOE/EH-231-011/0192

CERCLA Baseline Risk Assessment-Human Health Evaluation .DOEJEH-231-012/0692 Institutional Controls in a CERCLABaseline Risk Assessment DOEJEH-231-014/1292 Executive Order 12580: Superfund Implementation DOEJEH-231-015/0593 Preliminary Assessments (PAs) Under.CERCLA DOE/EH-231-016/0593 Natural Resource Damages Under CERCLA $\ldots \ldots \ldots \ldots \ldots \ldots \ldots$ DOE/EH-231-017/0693

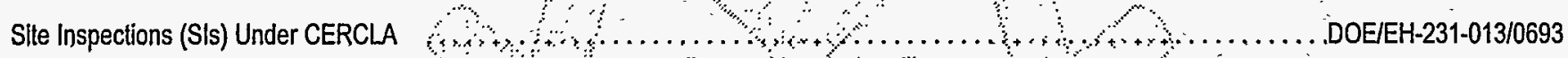
EPCRA Requirements .................. B. DOE/EH-231-018/1093 CERCLA Reporting Requirements DOE Occurrence Reportmg; and the totemengency Management System

Site Deletion from the National Prionieses list $\ldots+\cdots$

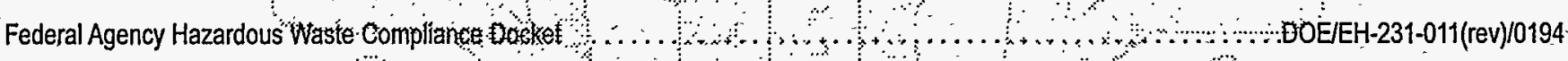

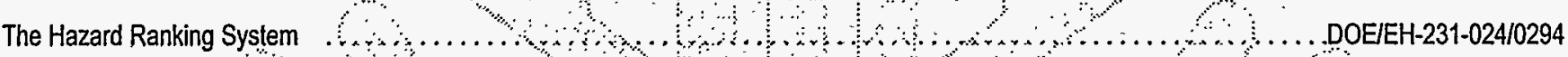
The Off-Site Rule $-\ldots$ and

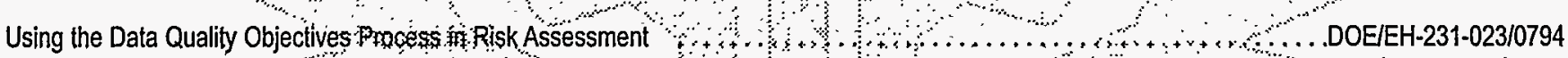
$\therefore \quad \therefore$

\section{CERCLA Regúfatory Bufletins}

Addition of Certain Chemicals - TRI Reporting final Rulè December 31, 1994 Transporting CERCLA Wastes Off-Site - Final Off-Site Rule December 31, 1994 National Oil and Hazardous Substances Pollution Contingency Plati- - Reviset fiule Issued July 31,1995

\section{RCRAICERCLA Gut̃dance Manuals}

RCRA/CERCLA Division Orientation Handbook :

Public Participation in Environmental Restoration Activities

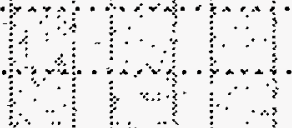

OSHA Training Requirements for Hazardous Waste Operations

Action Processes

A Comparison of the RCRA Corrective Action and CERCLARemediaf Action Frocesses

.DOE/EH-0187P, October 1991

DOEJEH-0221, November 1991

DOEEJEH-0227P, December 1991

Guide to Selecting Compliant Off-Site Hazardous Waste Treatment, Storage and Disposal Facilities

DOEIEH-0365; February 1994

RCRA Corrective Action \& CERCLA Remedial Action Reference Guide

.DOE/EH-0427, September 30, 1994 DOEEEH-0001, July 1994 


\section{RCRA/CERCLA Information Briefs}

Health Assessments and Other Activities of the Agency for Toxic Substances and Disease Registry .DOE/EH-231-006/0891

The Information Repository DOE/EH-231-009/1191

The Administrative Record DOE/EH-231:010/1191

RCRA and CERCLARequirements Associated with the Sale or Transfer of DOE Property DOEJEH-231-022/1193

Sireamlined Site Characterization Approach for Early Actions: Impact or Risk Assessment Data Requiements DOE/EH-231-025/1294

\section{TSCA Guidance Manuals}

Management of PCBs

DOÉJEH:0350, November 1993

\section{TSCA Information Briefs}

PCB.Manifesting, Tracking, and Disposal Requirements DOE/EH-231-001/1190 Disposal Requirements for PCB Waste The PCB Mark

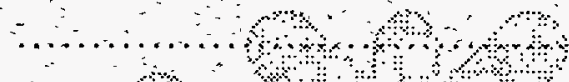
DOE/ËH-231-056/1294 PCB Recordkeeping and Reporting $\therefore \ldots \ldots$ ४. DOE/EH-231-057/1294 PCB Spill Response and Notification Renatmentiss

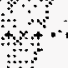
and $\therefore \ldots$ by DOE/EH-231-058/1294 be DOE/EH-231-059/1294 PCB Storage Requiremeits: on oross CortMarkats

Federal Environmental Inspections Hantabook a

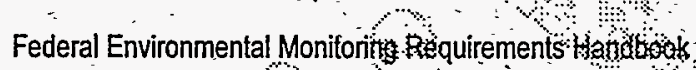
औ

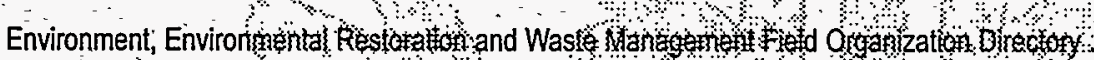

Federal Environmental Reporting exumements Handbook

3

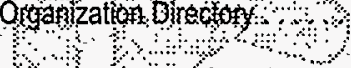

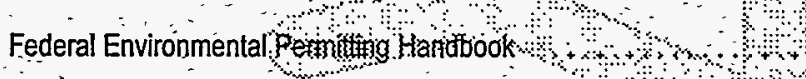

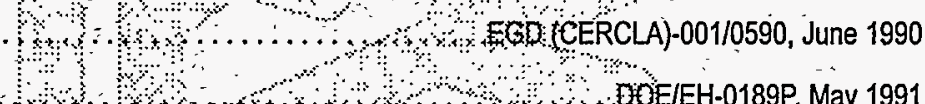

Glossary of CERCLA, RCRA, and TSCA-Relater Tertis amd Acronyths
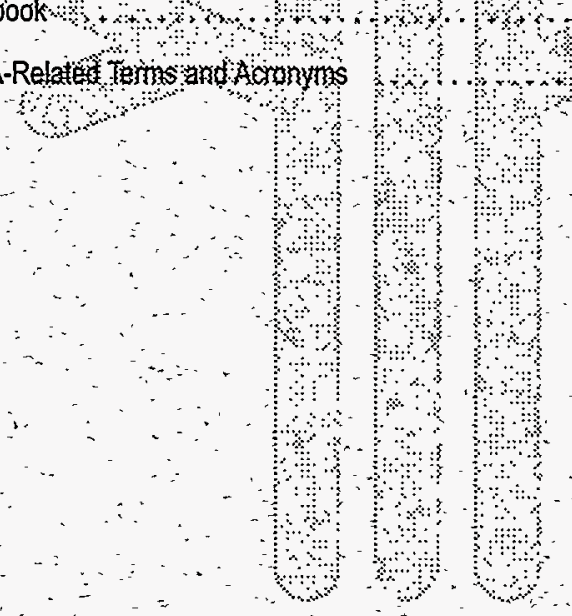

DOE/EH-231-060/1294


DOE/EH:0496, July 1995

DOE/EH-0347, October 1993 